\title{
Congenital Toxoplasmosis Infection in an Infant Born to an HIV-1-Infected Mother
}

\author{
Maria Letícia Santos Cruz, Claudete Araújo Cardoso, Mariza C. Saavedra, Eliane dos Santos and Tatiana Melino \\ Infectious Diseases Department, Hospital dos Servidores do Estado, Rio de Janeiro; Rio de Janeiro, RJ, Brazil

\begin{abstract}
We report the occurrence of congenital toxoplasmosis in an infant born to an HIV infected mother who had high anti-toxoplasma IgG and negative IgM at nine weeks of gestation. We briefly review available literature and discuss the possible mechanisms of transmission of congenital toxoplasmosis among HIV infected pregnant women. Key-Words: congenital toxoplasmosis, HIV-1 exposed infants, avidity test.
\end{abstract}

Medical literature about vertical transmission of Toxoplasma gondii among HIV-1-infected women is scarce. A study with 138 HIV-infected pregnant women in State University of New York-Brooklyn, where $20 \%$ had positive IgG for Toxoplasma, reported one case of congenital infection [1]. This report summarizes our first case of congenital toxoplasmosis diagnosed among $767 \mathrm{HIV}$-exposed infants followed at Infectious Diseases Department of Hospital dos Servidores do Estado, since 1996, in Rio de Janeiro, Brazil, where $74 \%$ of HIV-infected mothers had serologic evidence of anti-Toxoplasma gondii IgG during pregnancy.

\section{Case Report}

A 38-year-old Caucasian woman was diagnosed as HIVinfected during pregnancy and was referred for specialized care at the Infectious Diseases Department of Hospital dos Servidores do Estado, in Rio de Janeiro, in October 2005 at 15 weeks' gestation. Her history was positive for smoking (20 cigarettes/day) during pregnancy and occasional substance abuse (marijuana and cocaine) before pregnancy. This was her fifth pregnancy following four previous pregnancy interruptions. Her serology for HIV had been negative in 1992. Routine prenatal testing (September 2005) at nine weeks' gestation revealed anti-Toxoplasma IgG $>1,000$ $\mathrm{IU} / \mathrm{mL}$ and negative anti-Toxoplasma IgM by ELISA. Initial CD 4 count was 325 cells $/ \mathrm{mm}^{3}$ (17\% of total lymphocytes) and viral load was 87,000 copies/mL (4.94 log) by PRC Amplicor $^{\mathrm{R}}$. She was started on zidovudine, lamivudine, and vevirapine.

Three weeks after starting antiretroviral medication (ARV), she presented with a disseminated cutaneous rash. Nevirapine was discontinued and she received dextrochlorpheniramine maleate and prednisone ( $40 \mathrm{mg} / \mathrm{day})$ for three days. Subsequently, all ARV were withdrawn for two weeks and restarted when the rash had resolved. The new regimen included zidovudine, lamivudine and nelfinavir and was well tolerated. At 34 weeks' gestation she had a CD4 count of 908

Received on 20 August 2007; revised 15 October 2007.

Address for correspondence: Dr.Maria Letícia Santos Cruz. Rua Sacadura Cabral 178, Saúde, Hospital dos Servidores do Estado, Serviço de Doenças Infecciosas, $4^{\circ}$ andar anexo IV. Rio de Janeiro Zip code: 20221-903. Phone: (21) 2233 1505. Fax (21) 22331551

The Brazilian Journal of Infectious Diseases 2007;11(6):610-611. (C) 2007 by The Brazilian Journal of Infectious Diseases and Contexto Publishing. All rights reserved. cells $/ \mathrm{mm}^{3}$ (29\% of total lymphocytes) and viral load $<80$ RNA copies/mL.

Premature delivery occurred at 36 weeks' gestation by cesarean section. She gave birth to a 2,200 $\mathrm{g}$ female with Apgar scores of $9 / 9$ but respiratory distress during the first days of life led to routine laboratory screening that revealed antiToxoplasma $\mathrm{IgG}>2,500 \mathrm{IU} / \mathrm{mL}$ and a positive anti-Toxoplasma IgM by ELISA. She had a normal cerebral ultrasonographic study; the spinal fluid had no cells and mildly elevated protein $(256 \mathrm{mg} / \mathrm{dL})$. Funduscopy showed bilateral inactive scars of chorioretinitis. The child was discharged from the hospital at 15 days of life receiving sulfadiazine, pyrimethamine, and folic acid. During a follow-up visit anti-Toxoplasma serology by ELISA revealed IgG $>1,000 \mathrm{IU} / \mathrm{mL}$ (cut-off = 6.0 IU), IgM 5.92 $\mathrm{IU} / \mathrm{mL}$ (cut-off $=0.5$ ). Funduscopic reevaluation also confirmed the diagnosis of congenital toxoplasmosis.

Trimethoprim and sulfamethoxazole (TMP-SMX) for pneumocystis pneumonia (PCP) prophylaxis was not prescribed because of the potential increase of toxicity and the very low risk of HIV infection of the infant since the mother was started on antiretroviral treatment at 18 weeks' gestation and had undetectable viral load near delivery. The infant had two HIV RNA exams below detectable levels and was considered HIV-uninfected at the fifth month of life.

\section{Discussion}

In the state of Rio de Janeiro, Brazil, infection by Toxoplasma gondii in general population is reported to vary from 23 to $84 \%$, depending on socioeconomic status [2]. Coinfection of HIV-1 and Toxoplasma gondii is highly prevalent among pregnant women followed at HIV antenatal clinic at Hospital dos Servidores do Estado. Nevertheless this is the first case of congenital toxoplasmosis we have documented among women with reactive $\mathrm{IgG}$ during the first trimester of gestation. Unfortunately, in the present case, testing for IgG avidity was not performed and further serologic study of this mother who presented high IgG titers at nine weeks' gestation, was not carried out.

Transmission of Toxoplasma gondii to the fetus depends on occurrence of maternal parasitemia, cellular immunity and stage of placental development [3]. It occurs almost exclusively in women who acquired the infection during gestation but rare exceptions have been observed in three situations: 1) immunocompetent women infected shortly before conception [4]; 2) maternal reinfection during pregnancy [5] and 3) 
reactivation of latent infection in immunocompromised mothers $[1,6]$.

Placental colonization with Toxoplasma gondii is a potential source of infection of the infant long after maternal parasitemia has subsided and there can be a delay of many weeks between maternal and fetal infection [7]. Rare cases have demonstrated that infection in the three months before conception does not guarantee effective immunity against congenital Toxoplasma transmission and pregnant patients have been advised to receive spiramycin if lymphadenopathy had occurred during six months preceding pregnancy [7]. However, among HIV-infected women, lymphadenopathy caused by Toxoplasma gondii may not be recognized and the results of antibody titers may be difficult to interpret. It has been hypothesized that congenital infection acquired from a mother infected a few months before conception may have a more favorable prognosis because maternal immunity may delay transmission [4].

Normally seroconversion is indicative of acute infection and the absence of detectable specific IgM rules out recently acquired infection [8], but in the present case, such a high IgG titer at the ninth week of gestation despite a negative IgM ELISA should have raised the possibility of recently acquired infection. This is a situation where testing for $\operatorname{IgG}$ avidity may be useful in defining a therapeutic approach, since it enables to differentiate recently acquired from past infection. Depending on the method, IgG avidity test is useful to rule out infection acquired within the last four to five months [9] and is helpful during the first months of pregnancy when recent maternal infection will reveal low IgG avidity, identifying a situation of higher risk of fetal infection.

As other tools for early diagnosis, detection of parasite in amniotic fluid can be accomplished by in vitro culture, animal inoculation and PCR [10]. PCR for detection of T. gondii has revolutionized prenatal diagnosis, avoiding the use of more invasive procedures on the fetus, thanks to its sensitivity, specificity and rapidity [11]. There are also marked differences in PCR sensitivity depending on gestational age at the time of amniocentesis. Furthermore, PCR on amniotic fluid is not recommended for HIV-infected women, because of the risk of transmitting the HIV virus to the fetus during the procedure [11].

Congenital transmission of toxoplasmosis due to reactivation of latent infection during pregnancy occurs in mothers with very low CD4 cell counts [1] or in the presence of other immunological disorders [8]. In this case the mother had relatively preserved immunity since the beginning of follow-up and an improving CD4 cell count after initiation of antiretroviral medication. It is very difficult to evaluate the impact on immunity of $40 \mathrm{mg}$ prednisone/day for three days prescribed to treat a reaction to nevirapine during the second trimester of pregnancy. There are reports of congenital toxoplasmosis in infants born to patients with systemic lupus erythematosus who used moderate doses of prednisone during pregnancy [3].

This case underscores the need for special attention to maternal titers of anti-Toxoplasma antibody during HIV prenatal care. The potential reactivation effect of the short course of prednisone to treat the nevirapine reaction must be considered, but we could not exclude recent infection, reinfection, or reactivation due to the mother's initially low CD4 cell count.

\section{References}

1. Minkoff H., Remington J.S., Holman S., et al. Vertical Transmission of toxoplasma by human immunodeficiency virus-infected women. Am J Obstet Gynecol 1997;176(3):555-9.

2. Bahia-Oliveira L.M., Jones J.L., Azevedo-Silva J., et al. Highly endemic, waterborne toxoplasmosis in north Rio de Janeiro State, Brazil. Emerging Infectious Diseases 2003;9(1):55-62.

3. Desmonts G., Couvreur J., Thulliez P. Toxoplasmose congénitale - Cinq cas de transmission à l'enfant d'une infection maternelle antérieure à la grossesse. Presse Méd 1990;19(31):1445-9.

4. Vogel N., Kirisits M., Michael E., et al. Congenital toxoplasmosis transmitted from an immunologically competent mother infected before conception. CID 1996;23:1055-60.

5. Gavinet M.F., Robert F., Firtion G., et al. Congenital toxoplasmosis due to maternal reinfection during pregnancy. J Clin Microb 1997;35(5):1276-7

6. Montoya J.G, Kovacs J.A. Toxoplasma gondi. In: Mandell G.L., Bennett J.E., Dolin R. Principles and Practice of Infectious Deseases, $6^{\text {th }}$ edition, by Elsevier, Churchill, Livingstone, 2005.

7. Remington J.S., McLeod R., Thulliez P., Desmonts G. Toxoplasmosis. In: Remington \& Klein, Wilson \& Baker. Infectious Diseases of the Fetus and Newborn Infant. Philadelphia: Elsevier Saunders, 2006.

8. Montoya J.G., Liesenfeld O. Toxoplasmosis. The Lancet 2004;363:1965-75

9. Remington J.S., Thulliez P., Montoya J.G. Recent developments for diagnosis of tozoplasmosis. J Clin Microbiol 2004;42:941-5.

10. Morussi Reis M., Tessaro M.M., D’Azevedo P.A. ToxoplasmaIgM and IgG-avidity in single samples from areas with a high infection rate can determine the risk of mother-to-child transmission. Rev. Inst. Med. Trop. S. Paulo 2006;48(2).

11. Montoya J.G. Laboratory of Toxoplasma gondii. Infection and Toxoplasmosis. J Infec Dis 2002;185(suppl.1):s73-82. 